\title{
Impacto de los Intermediarios en los Sistemas de Innovación
}

\author{
Walter Lugo Ruiz Castañeda ${ }^{* 1}$, Santiago Quintero Ramírez ${ }^{2}$, Jorge Robledo Velásquez ${ }^{3}$
}

Resumen: La perspectiva sistémica ha sido aplicada extensamente al estudio de la innovación, dada la existencia de múltiples agentes heterogéneos cuya interacción permite la generación, difusión y uso del conocimiento. Sin embargo, tal interacción presenta dificultades por las brechas existentes entre los agentes, siendo los intermediarios los responsables de construir puentes y facilitar la vinculación. No obstante, analizar el impacto de los intermediarios no es fácil, principalmente por el problema de atribución, que reduce muchas aproximaciones a fotografías carentes de análisis dinámico y longitudinal. Este trabajo propone superar tales limitaciones mediante simulación basada en agentes, la cual ayuda a ampliar el entendimiento del fenómeno de la intermediación en los sistemas de innovación y su impacto en el desempeño.

Palabras claves: Intermediarios de innovación; sistemas de innovación; modelación basada en agentes; capacidades de innovación; costos de transacción

Title: Impact of Intermediaries in Innovation Systems

Abstract: The systemic approach has been widely applied to the study of innovation, given the existence of multiple heterogeneous agents whose interaction allows the generation, diffusion and use of knowledge. However, such interaction presents difficulties because there are gaps between agents, being the intermediaries responsible for building bridges and facilitating linkage. Nevertheless, analyzing the impact of intermediaries is not easy, mainly because the problem of attribution, where different approaches are nothing more than a photography that lacks a dynamic and longitudinal analysis. This work aims to overcome such limitations by agent-based simulation, which helps broaden understanding of the phenomenon of intermediation in innovation systems and their impact on performance.

Keywords: innovation intermediaries, innovation systems, agent-based modeling, innovation capabilities, transaction cost

Submitted: March 29 $2016 /$ Approved: $\quad$ Approved: June $9^{\text {th }} 2016$

\section{Introducción}

El relacionamiento de agentes heterogéneos que conforman los sistemas de innovación no es sencillo, debido a la existencia de brechas de diferentes dimensiones entre ellos (Parjanen, Melkas, \& Outila, 2011), ocasionando unos altos costos de transacción (CT) (Batterink, Wubben, Klerkx, \& Omta, 2010). Son los intermediarios quienes encaran esta dificultad mediante la construcción de puentes entre agentes distantes y la generación de confianza, favoreciendo el relacionamiento que permite el establecimiento de un sistema de innovación. Como expresan Ruiz y Robledo (2013), las diferentes perspectivas que han abordado el fenómeno de la intermediación, reconocieron la necesidad de precisar el impacto de los intermediarios en el sistema en el que están inmersos; del mismo modo, plantearon que tal evaluación es un problema complejo, dadas las dificultades que hay en la atribución de su aporte al sistema de innovación. Se encuentra entonces que, para una correcta valoración de tal aporte se requiere un análisis dinámico y longitudinal que describa el desempeño y co-evolución de los diferentes agentes que conforman el sistema de innovación, siendo esto fundamental para poseer elementos que evidencien la contribución de los intermediarios y se puedan tomar decisiones con respecto a estos agentes (Ruiz \& Robledo, 2013).
Desde la postguerra se han desarrollado modelos orientados a ayudar al entendimiento del proceso de innovación, desde visiones reduccionistas como los enfoques lineales de primera generación hasta los modelos sistémicos de quinta generación (Rothwell, 1994). Estos últimos entienden el proceso de innovación como sistémico y resaltan la importancia del aprendizaje. Sin embargo, los mecanismos responsables de la conformación de los sistemas de innovación no son fáciles de comprender, principalmente por la complejidad de los procesos dinámicos y por la heterogeneidad de los actores que intervienen (Gilbert, Pyka, \& Ahrweiler, 2001). Por lo anterior, los sistemas de innovación se pueden considerar como Sistemas Complejos Adaptables (SCA). Estos últimos se conciben como un arreglo de agentes interactuantes descritos por reglas, las cuales cambian al acumular experiencia (Holland, 2004). Estos SCA son representarlos recurrentemente a través de la Modelación Basada en Agentes (MBA), por ser una herramienta potente para obtener información de la dinámica de sistemas que son conformados por agentes heterogéneos y el relacionamiento entre ellos tiene sus características propias (Rahmandad \& Sterman, 2008). Justificando la adopción de esta aproximación para afrontar el problema de análisis del impacto de los intermediarios en los sistemas de innovación.

(1) Centro de Desarrollo Empresarial, Universidad Pontificia Bolivariana, Medellín, Colombia,

(2) Ingeniería Industrial, Universidad Pontificia Bolivariana, Medellín, Colombia.

(3) Dpto. de Ingeniería de la Organización, Universidad Nacional de Colombia, Sede Medellín, Colombia.

*Corresponding author: walterlugo.ruiz@upb.edu.co 
A continuación se plantean unos supuestos principales, con los cuales se elabora el modelo conceptual y la lógica de la simulación. Luego se aplica el modelo de simulación mediante la parametrización del modelo y la simulación de escenarios que permitan hacer experimentos con los intermediarios, con el fin de analizar su impacto en el desempeño del sistema de innovación. Para finalizar, se presentan las conclusiones y unas recomendaciones de trabajo futuro.

\section{Formulación del modelo}

\section{Supuestos fundamentales}

Para analizar el impacto de los intermediarios en los sistemas de innovación a través de un modelo de simulación, se debe tener en cuenta que estos sistemas emergen de la interacción entre agentes heterogéneos (Edquist, 1997). Dicho modelo debe de permitir realizar experimentos con los agentes intermediarios para poder analizar su impacto en el desempeño del sistema.

Las capacidades que requiere el sistema de innovación para realizar sus funciones de generar, difundir y usar conocimiento y tecnología están distribuidas entre los diferentes agentes que lo conforman. Estas capacidades han sido clasificadas por varios autores y se les ha dado la connotación de capacidades tecnológicas, de innovación y/o de innovación tecnológica (Kim, 1997; Ernst, Mytelka, \& Ganiatsos, 1998; Guan \& Ma, 2003; Wang, Lu, \& Chen, 2009). En la Tabla 1 se pueden ver cómo cada capacidad de innovación es asignadas a una función de los sistemas de innovación:

Tabla 1. Asignación de capacidades de innovación a las funciones de los sistemas de innovación

\begin{tabular}{|c|c|c|}
\hline Función & Capacidad & Aplicación \\
\hline \multirow{2}{*}{$\begin{array}{l}\text { Generación de conocimiento y } \\
\text { tecnología }\end{array}$} & Investigación & Generar y adaptar conocimiento y tecnologías. \\
\hline & Desarrollo & $\begin{array}{l}\text { Desarrollar experimentalmente productos, procesos, métodos de mercadeo y formas de } \\
\text { organización }\end{array}$ \\
\hline \multirow{2}{*}{$\begin{array}{l}\text { Difusión de conocimiento y } \\
\text { tecnología }\end{array}$} & Difusión & Capturar resultados de I+D y tecnologías y aprovechar sus beneficios. \\
\hline & Vinculación & $\begin{array}{l}\text { Realizar transferencia de tecnología interna, entre agentes y la infraestructura local de } \\
\text { ciencia y tecnología }\end{array}$ \\
\hline \multirow{2}{*}{ Uso de conocimiento y tecnología } & Producción & $\begin{array}{l}\text { Operar y mantener su infraestructura productiva de forma eficiente, así como adaptar y } \\
\text { mejorar la tecnología de producción existente. }\end{array}$ \\
\hline & Mercadeo de la innovación & $\begin{array}{l}\text { Identificar necesidades presentes y futuras del mercado, desarrollar nuevos productos, } \\
\text { establecer canales de distribución, prestar servicios al cliente y publicitar la innovación. }\end{array}$ \\
\hline
\end{tabular}

Las capacidades de innovación con que cuentan los agentes los caracteriza de la siguiente forma: los explotadores poseen capacidades de producción y/o mercadeo de la innovación, los intermediarios tienen capacidades de difusión y/o vinculación, y los exploradores ostentan capacidades de investigación y/o desarrollo. Estos agentes se pueden considerar como especializados en una función de los sistemas de innovación. Sin embargo, en el modelo propuesto se permite que los agentes puedan ejercer varias funciones, los cuales se clasifican como: introductores o porteros, que tienen capacidades para explotar e intermediar; representantes o gestores, que pueden explorar e intermediar; integrados, que consiguen explorar, intermediar y explotar; ambidiestros, que exploran y explotan, y los de desarrollo incipiente, que no se distinguen por una alta capacidad en ninguna función de los sistemas de innovación.

Para que los agentes se puedan considerar como competentes, sus capacidades deben de ser validadas por un entorno competitivo. En el modelo propuesto, este entorno es representado por necesidades que requieren ser satisfechas por los agentes, quienes mediante sus capacidades mínimas deben de suplir los atributos de cada necesidad, dándole al modelo un comportamiento market pull. Estos atributos se manifiestan en vectores que representan innovaciones, los cuales se denominan oportunidades de innovación (OI). Los valores en cada posición del vector de atributos determinan las necesidades de cada oportunidad de innovación.
Para finalizar, una consideración significativa es la de reconocer la dificultad en la interacción entre agentes producto de la brecha que se genera por su heterogeneidad, especialmente entre exploradores y explotadores, causando altos CT que influyen en el desempeño de los agentes y el sistema. Estos CT dependen de la tipología de los agentes que están interactuando, sustentándose esta afirmación en el planteamiento de Williamson (1985), quien sostiene que siempre que los activos son específicos en un grado no trivial, en el caso del modelo las capacidades de innovación que hacen que los agentes competidores sean heterogéneos, hay un incremento en la incertidumbre, la cual ocasiona que las brechas contractuales sean mayores. En esta dirección, los agentes intermediarios que poseen altas capacidades de difusión y vinculación, generan confianza y, por ende, disminuyen los CT en las interacciones en las que están involucrados. De forma similar, los agentes introductores o porteros, representantes o gestores e integrados, que también cuentan con capacidades de difusión y vinculación, pueden generar CT bajos o medios dependiendo de los agentes con que estén interactuando. Por último, los agentes que no poseen capacidades de difusión o de vinculación como los exploradores, explotadores y agentes de desarrollo incipiente, generan unos CT altos en sus interacciones. 


\section{Modelo conceptual}

Los supuestos que rigen el modelo se presentan en la Figura 1, donde se observan los diferentes tipos de agentes según sus capacidades, ejemplos de vectores de capacidades de cada tipo de agente, la función que cumplen en el sistema de innovación según sus capacidades, los diferentes vínculos que se pueden generar, el CT que se asigna a cada vínculo, y cómo esta relación contingente entre agentes puede aprovechar OI que están presentes en el entorno competitivo mediante la innovación en producto, proceso, método de mercadeo u organizacional.

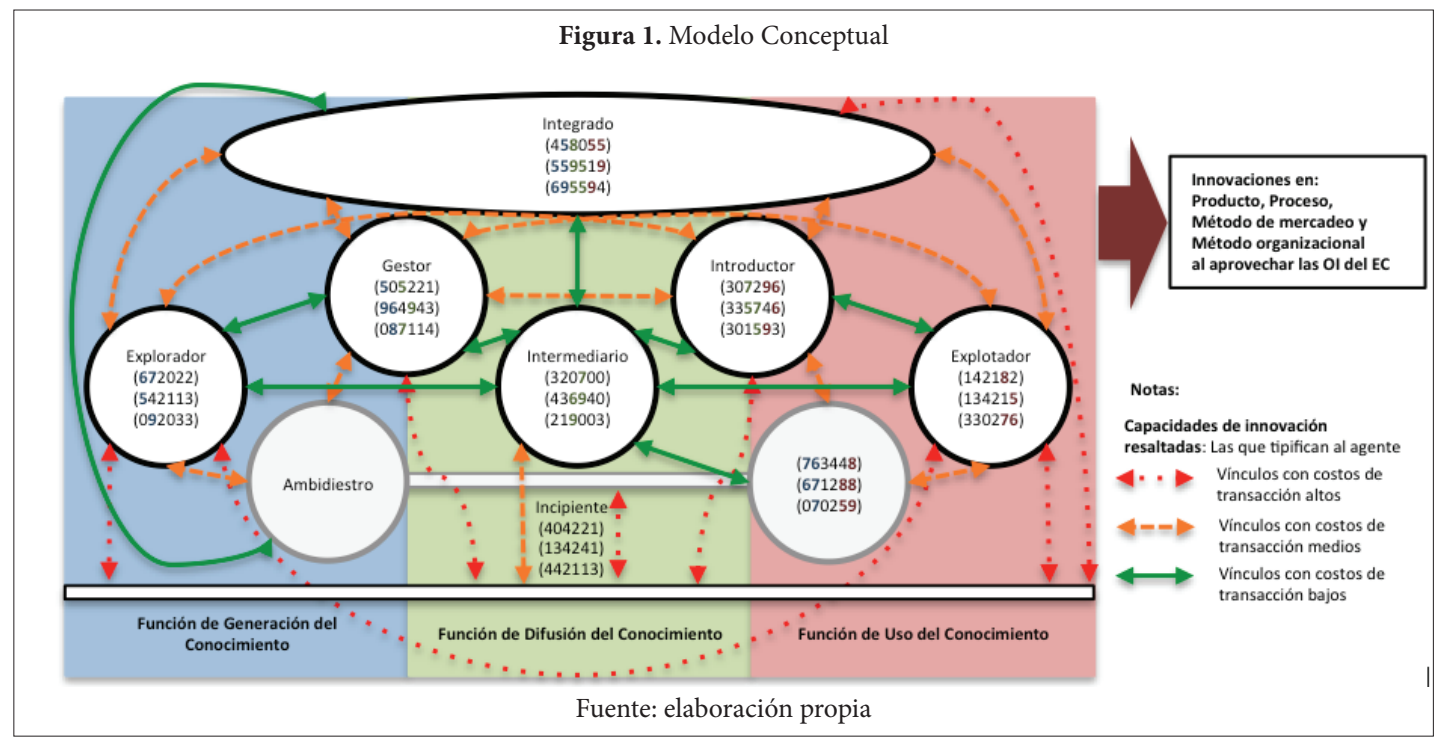

\section{Lógica del modelo de simulación}

Las reglas de decisión del modelo de simulación se pueden apreciar en el diagrama de flujo presentado en la Figura 2, donde la localización juega un papel fundamental al ser la primera regla de búsqueda de agentes. Luego, la complementariedad en las capacidades es la regla que define si se realiza el vínculo o no. Esta búsqueda se rige por ir de la explotación a la exploración o de derecha a izquierda en el vector de atributos de las $\mathrm{OI}(\mathrm{dDaI})$. Cada vínculo entre agentes según su tipo, determina el CT. Cuando una oportunidad de innovación es aprovechada, esta reparte sus beneficios entre los agentes o agente que suplen sus atributos con sus capacidades, de acuerdo a la magnitud de cada atributo y el ciclo de vida de la oportunidad de innovación. Estos beneficios se suman al stock de excedentes de cada agente por período, al cual se le restan los costos de mantener sus capacidades y los CT de cada uno de sus vínculos. Los agentes que disminuyan su stock de excedentes hasta cero desaparecerán del sistema. Los agentes que suplan OI aprenden acumulando las capacidades que utilizaron y des-acumulan las que no mediante el des-aprendizaje.

\section{Aplicación del modelo de simulación}

Los valores iniciales de los parámetros del modelo y la lógica para definirlos se pueden apreciar en la Tabla 2. Ahora, para la elección de los escenarios se tiene en cuenta el planteamiento de Holland (2004, p. 55), sobre que "todos los SCA tienen puntos de apalancamiento, en donde pequeñas adiciones producen grandes cambios dirigidos"; por ello, se analiza al intermediario como posible punto de apalancamiento que genere un desempeño diferenciado del sistema de innovación.
Figura 2. Diagrama de flujo de la lógica del modelo

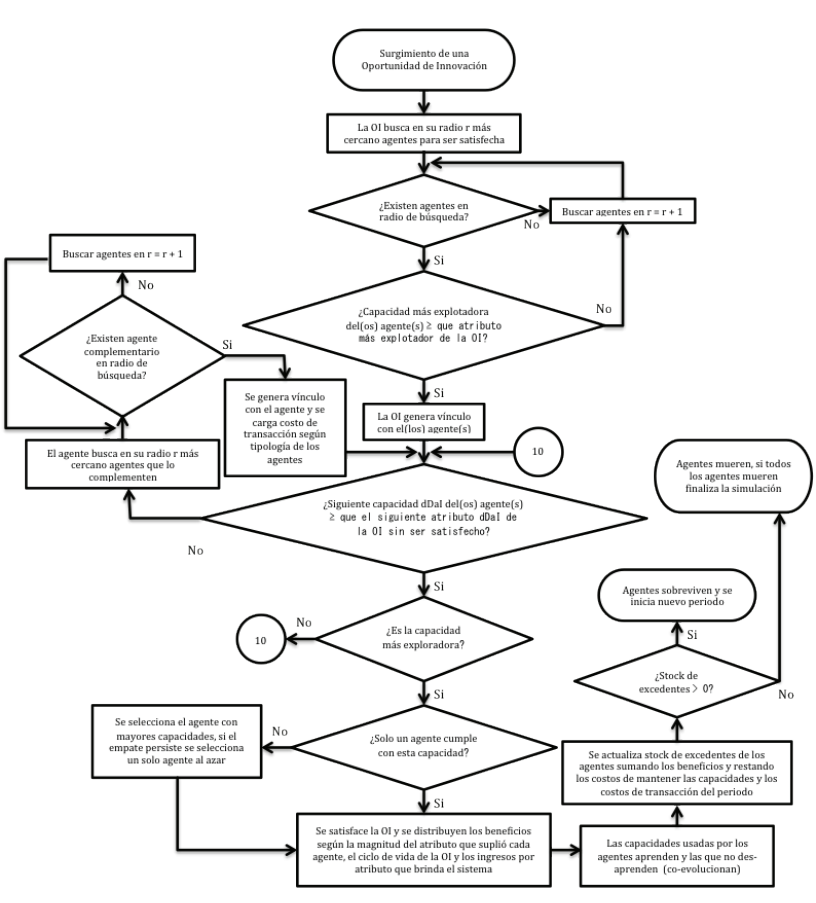

Fuente: elaboración propia 
Las simulaciones se realizan en el programa NetLogo 5.1.0. Cabe anotar que estas simulaciones son consideradas como exploraciones de micromundos, o mundos simplificados y manipulables (Resnick,
2001), por lo que se puede hablar más de exploración de micromundos que de simulaciones de la realidad (Resnick, 2001).

Tabla 2. Valores de los parámetros del modelo

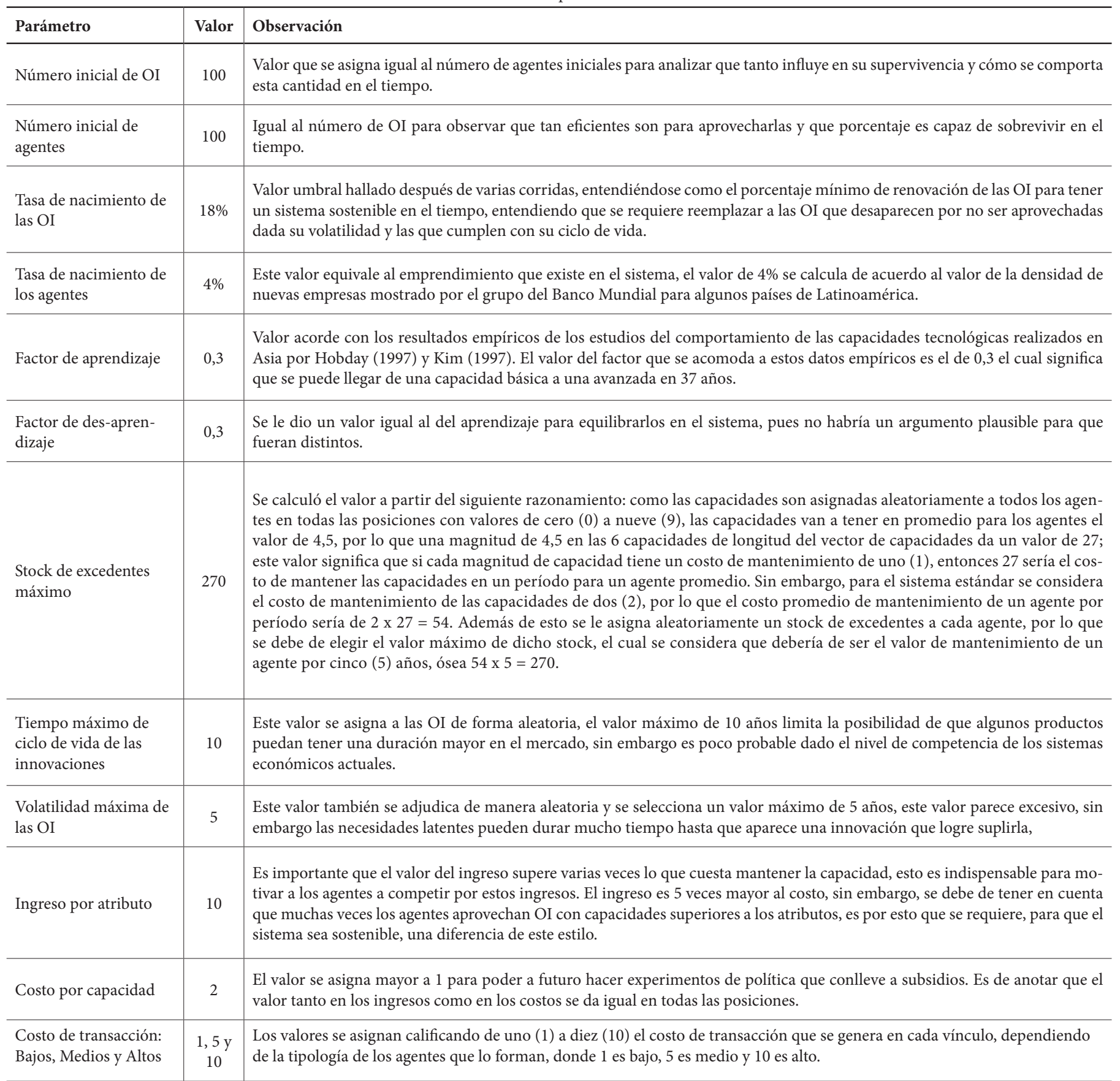


Considerando lo anterior, se plantean los siguientes escenarios:

\section{Escenario problema $(\mathrm{A})$}

Como ya se mencionó, las capacidades con las que deben contar los agentes para realizar la función de intermediación en los sistemas de innovación son las de difusión y vinculación. Por ello, un escenario problemático para que exista realmente un sistema de innovación, es que ningún agente posea capacidades de difusión y de vinculación.

En este escenario no existe intermediario, ni tampoco se les da la opción a los otros agentes para que asuman la función del intermediario. Esto se logra asignando un nivel de cero a las capacidades de difusión y de vinculación de todos los agentes del micromundo.

\section{Escenario problema con un intermediario (B)}

Siguiendo la recomendación de Holland (2004) de buscar identificar puntos de apalancamiento, se parte del escenario problema y se le adiciona un único agente intermediario con unas capacidades medias (valor de cinco) de difusión y vinculación, con el fin de analizar qué impacto genera este agente en el desempeño del micromundo.

Es de notar que para todos los escenarios, las magnitudes de las posiciones de los vectores de las OI del entorno competitivo se asignan de forma aleatoria de cero (0) a nueve (9).

\section{Escenario sin intermediarios pero con capacidades de difusión y} vinculación distribuidas en todo el micromundo (C)

En este escenario se asigna a todos los agentes del micromundo capacidades de difusión y vinculación de forma aleatoria: pudiendo carecer de ellas (valor de cero), o tener niveles incipientes (de uno a tres), o niveles medios (de cuatro a seis), o niveles avanzados (de siete a nueve), en cualquiera de las dos capacidades encargadas de las funciones de difusión. Sin embargo, se elimina la opción de que existan agentes intermediarios, o sea, aquellos que por sus capacidades se especializan solo en la función de difusión de conocimiento y tecnologías.

A diferencia de los escenarios anteriores, en este interactúan en el micromundo agentes del tipo introductores o porteros, representantes o gestores, e integrados.

Escenario con intermediarios y con capacidades de difusión y vinculación en todo el micromundo (D)

Se considera que este es el escenario 'ideal' o 'normal', donde puede existir todo tipo de agentes y las capacidades se asignan de forma aleatoria para todos ellos. En este micromundo todos los agentes tienen la posibilidad de generar, difundir y usar conocimiento y tecnología para aprovechar las OI del entorno competitivo.

La diferencia de este escenario con el anterior es el permitir que existan agentes que por su tipología se consideran intermediarios, lo cual se hace con el mismo fin del escenario dos de identificar puntos de apalancamiento en el desempeño del micromundo.

\section{Resultados y análisis de los escenarios}

A continuación se comparan los resultados de las simulaciones de los cuatro escenarios, donde se puede ver la evolución de las principales variables del modelo (ver Figura 3). El tiempo de simulación es de 25 años, lapso suficiente para identificar las tendencias en el comportamiento de cada uno de los escenarios. A los datos obtenidos de las simulaciones se les realizó un análisis estadístico, donde primero se analizó si había diferencia significativa entre los cuatros escenarios, para luego aplicar la prueba de Tukey para identificar esas diferencias entre los comportamientos de cada escenario.

Figura 3. Evolución de las principales variables del modelo para los cuatro escenarios de simulación
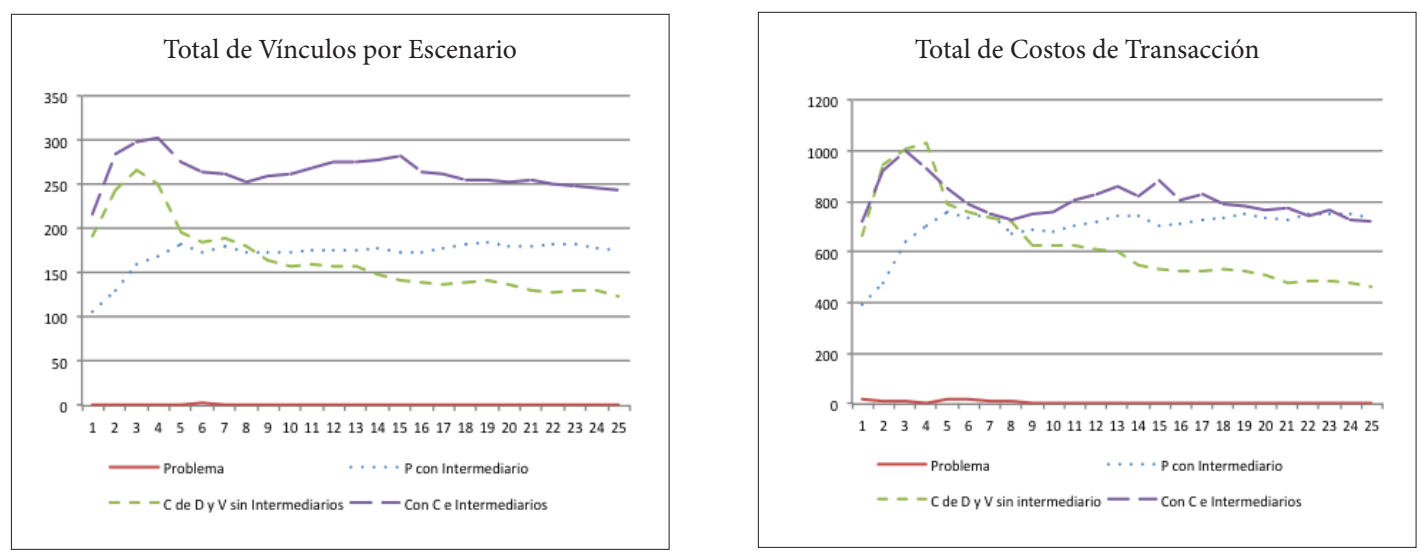

ISSN: 0718-2724. (http://jotmi.org) 

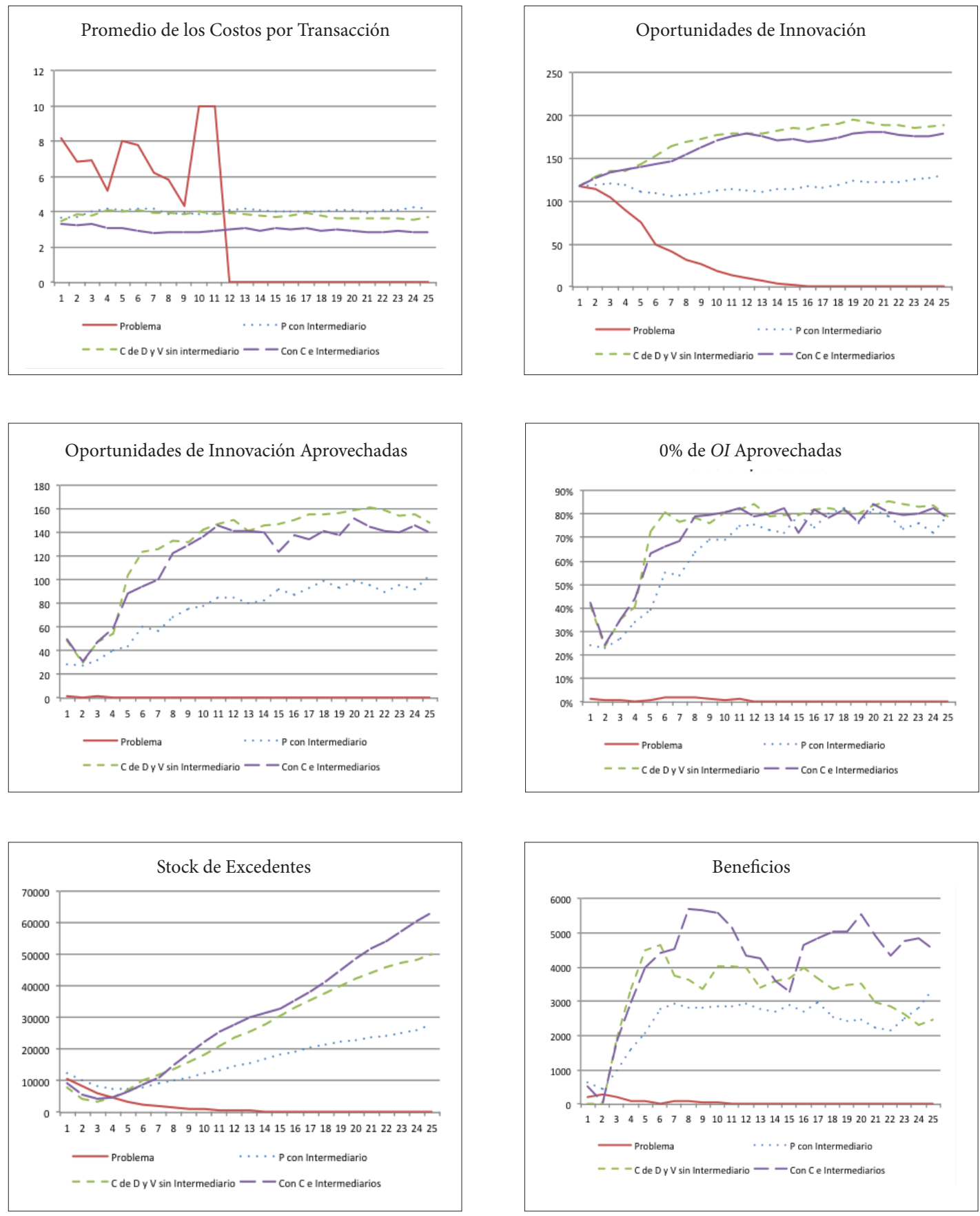

Fuente: Elaboración propia basado en los resultados de las simulaciones en NetLogo 5.1.0

El total de vínculos o transacciones se puede interpretar como si evidentemente existe o no un sistema. Esto se puede afirmar puesto que, como manifiesta Bertalanffy (1968) en su Teoría General de Sistemas, para que haya un sistema las unidades deben de estar en interacción.

La gran diferencia en el comportamiento de los escenarios A y B se tenía prevista, teniendo en cuenta lo manifestado por Klerkx y Leeuwis (2008; 2009) y Batterink et al. (2010) donde los intermediarios se consideran como formadores y orquestadores del sistema de innovación.
La diferencia en los dos comportamientos es significativa, aunque el único cambio realizado es la introducción de un agente intermediario con unas capacidades medias de difusión y vinculación, permitiendo esto identificar un punto de apalancamiento. Ahora, al comparar los escenarios $\mathrm{C}$ y D, NO se encuentra diferencia significativa. Teniendo en cuenta esto último, NO se puede hablar de un punto de apalancamiento cuando se introducen intermediarios en un micromundo donde ya existen capacidades de difusión y vinculación; muy diferente a lo observado entre el escenario A y B. 
El actuar del intermediario en el caso del escenario B y las capacidades de difusión y vinculación del escenario $\mathrm{C}$ y la combinación de estos elementos en el escenario D, mantiene unos CT promedio por vínculo similares; demostrando cómo las capacidades de difusión y vinculación, ya sea aportadas por intermediarios o por otro tipo de agentes, son importantes para reducir los CT presentes en los sistemas de innovación.

El comportamiento observado en la Figuras 3, respecto al número de OI del entorno competitivo y al número de OI aprovechadas por los agentes competidores, tiene mucha relación, pues mientras más OI sean aprovechadas, puede aparecer una mayor cantidad de ellas en el micromundo, mostrando cómo los ambientes más innovadores generan una mayor dinámica. El número y porcentaje de OI aprovechadas por los agentes competidores depende mucho de las capacidades que tengan los agentes del micromundo para generar, difundir y usar conocimiento y tecnología, así: en el caso del escenario A, donde los agentes no cuentan ni con capacidades de difusión y vinculación, se distingue cómo los agentes no logran aprovechar las OI y poco a poco estas van desapareciendo debido a su volatilidad. Mientras que al agregarle un intermediario en el escenario $\mathrm{B}$, el número de OI se logra mantener estable en el mundo, pues los agentes logran aprovecharlas a través de la interacción y el aprendizaje de las capacidades que están utilizando. Los escenarios C y D presentan unos comportamientos muy similares; esto se puede considerar un comportamiento contraintuitivo, ya que se esperaba que el escenario D, por el actuar de los intermediarios, tuviera mejores resultados que el escenario C. En síntesis, como en el caso de vínculos, en las OI también se puede notar el efecto de apalancamiento que tiene el instalar un intermediario en un micromundo que no posee capacidades de difusión y vinculación.

El análisis del desempeño económico de los escenarios se hace a partir del comportamiento del stock de excedentes y beneficios (Figura 3). Estos comportamientos van de la mano de la cantidad de OI aprovechadas, pues son estas las que otorgan los beneficios a los agentes del micromundo. El escenario A obtiene unos beneficios muy pobres en comparación con los otros escenarios; estos beneficios aportan poco al stock de excedentes, que se va viendo menguado por los costos de mantenimiento. Ahora, al agregarle un intermediario a ese escenario problema (escenario B), se aprecia un comportamiento diferente, donde los beneficios y el stock de excedentes van aumentando, se estabilizan y mantienen en el tiempo, puesto que los agentes van especializándose por la acumulación de capacidades que propicia el aprendizaje; sin embargo, los resultados no alcanzan a ser tan buenos como cuando existen más capacidades de difusión y vinculación en el micromundo (escenarios $\mathrm{C}$ y D), pero sí son mucho mejores que en el escenario A.

Los escenarios C y D, con capacidades de difusión y vinculación distribuidas entre los agentes del micromundo, presentan mejores resultados en los beneficios y stock de excedentes, lo cual se puede explicar por su éxito en el aprovechamiento de OI, comportamiento provocado por la cantidad de vínculos entre agentes, por la acumulación de capacidades y por la co-especialización de los agentes. Esta conducta genera unos mejores resultados a la hora de aprovechar las OI, permitiendo que los agentes logren aumentar su stock de excedentes gracias a su desempeño innovador. Sin embargo, estos escenarios no presentan diferencias significativas en el stock de excedentes, por lo que el impacto del intermediario en este indicador del desempeño económico es poco significativo.

En las simulaciones de los escenarios se pudo apreciar la generación de un contexto dinámico caracterizado por la co-evolución y la autoorganización; esto se puede ver en la Figura 3, especialmente al inicio de las simulaciones de los micromundos, donde los agentes inician sus relaciones y empiezan a co-evolucionar y co-especializarse gracias al aprendizaje que se da vía interacción con otros agentes complementarios y al uso de sus capacidades, que les permiten acumularlas y des-acumularlas. Ese comportamiento caótico al inicio de las simulaciones puede no corresponder a un sistema real, pues aunque se ha tratado de ajustar los parámetros del micromundo, ningún sistema real empieza en $\mathrm{t}=0$ de una manera puramente aleatoria. Posteriormente, la propensión en los comportamientos se va volviendo más persistente, reflejando la auto-organización que se da en los SCA, que los lleva a mostrar el orden oculto del que habla Holland (2004).

El escenario B observado en la Figura 3 es bastante revelador, pues en este el intermediario juega un papel donde aporta sus capacidades para que se dé el puente entre los agentes extremos facilitando la difusión. También se tiene en cuenta en todos los escenarios que el intermediario no pretende reemplazar las relaciones que ya se han creado entre los agentes del micromundo; sin embargo, no todos los agentes tienen las mismas capacidades de difusión y vinculación; por lo tanto, el intermediario actúa como facilitador, especialmente entre los agentes que presentan más debilidades en estos sentidos, tal cual como lo manifiesta Göktepe (2006).

Una contribución importante del intermediario que se puede observar, especialmente en el escenario B, reconocida por Klerkx y Leeuwis (2008), es su construcción de capacidades en los demandantes y proveedores de conocimiento para cooperar en el proceso de innovación; dicha labor también es realizada en los escenarios C y D por los agentes que poseen capacidades de difusión y vinculación. De igual forma, se identifica en los escenarios B, C y D que los intermediarios contribuyen reduciendo los CT promedio de los vínculos involucrados en el intercambio de conocimientos, aporte reconocido por Dyer y Singh (1998) para los intermediarios, el cual se visualiza especialmente en el escenario B. En el caso del escenario B, que transforma fundamentalmente al escenario A al agregar un intermediario, se podría usar la conclusión de Boon, Moors, Nahuis y Vandeberg (2008), quienes plantean que la interacción entre usuarios y productores de conocimiento organizados a través de intermediarios, parece ser la herramienta más importante para la articulación de la demanda de conocimiento y el aprendizaje interactivo.

En cuanto a la diferencia entre ingresar un intermediario a un micromundo donde no existen capacidades de difusión y vinculación y uno en el que sí, como en los casos de los escenarios B y D, la diferencia en los resultados confirma lo expresado por Konttinen, Nieminen y Suvinen (2010), cuando dicen que se justifica la intervención de 
políticas para apoyar la conformación de intermediarios, siempre y cuando la relación Universidad-Empresa-Estado no esté funcionando bien; por ello, en el caso del escenario D, son escasas las contribuciones que los intermediarios aportan al comportamiento al compararlo con el escenario C; siendo muy diferente e impactante el agregar un intermediario en el escenario A. Sin embargo, como se asignaron capacidades de todo tipo en los escenarios C y D, así como en las capacidades que hacen parte de las funciones de generación y uso de conocimiento en los escenarios A y B, no se puede perder de vista lo expresado por Van Lente, Hekkert, Smits y Van Waveren (2003), cuando reconocen que aunque es útil e incluso necesario el rol del intermediario, no es suficiente para el buen desempeño del sistema, el cual sigue dependiendo de la composición del mismo o, en otras palabras, de los actores empresariales y científicos que lo componen.

Con las variables de salida de los escenarios se pretende analizar el impacto de los intermediarios en el desempeño del Sistema de Innovación, como se hizo en este apartado. Sin embargo, hay unos comportamientos emergentes que vale la pena resaltar. En primer lugar, no solo el intermediario impacta en el desempeño del sistema de innovación, sino que permite la conformación y orquestación del mismo como se pudo apreciar en el escenario B, mostrando el gran impacto que tiene el intermediario en lugares donde no se ha logrado establecer un sistema de innovación por las escasas capacidades de difusión y vinculación que existe en los agentes, lo que ha conformado unas brechas que son superadas gracias al intermediario. En segundo lugar, el impacto que genera el intermediario en el sistema de innovación, también depende de las capacidades de los agentes que conforman el sistema; por ejemplo, como se pudo observar al agregar intermediarios en sistemas que ya cuentan con capacidades de difusión y vinculación y que no poseen unas brechas tan grandes entre los agentes, siendo su impacto no tan notorio como cuando se instala en lugares donde los agentes no cuentan con estas características.

\section{Conclusiones y trabajo futuro}

El modelo permite zanjar el problema de atribución del intermediario manifestado en la literatura sobre intermediarios de innovación, mediante un análisis comparativo, dinámico y longitudinal, que admite analizar el impacto de los intermediarios en el desempeño y conformación del sistema de innovación. Al comparar el comportamiento de los cuatro escenarios en el tiempo se puede observar que los intermediarios tienen un impacto diferente en el desempeño del sistema de innovación de acuerdo a las capacidades con que cuenten los agentes con los que va a interactuar. En el caso de un lugar donde no se ha formado un sistema de innovación, donde existen brechas entre los agentes que generan y usan conocimiento, y donde las capacidades de difusión y vinculación con las que cuentan los agentes del sistema son insuficientes, el intermediario actúa como un punto de apalancamiento que permite la formación del sistema de innovación e impacta en el desempeño innovador y económico del mismo. Mientras que, en el caso de un lugar donde los agentes que van a conformar el sistema poseen capacidades de difusión y vinculación y, por ende, menos brechas entre algunos agentes, el adicionar agentes intermediarios, aunque en algunos casos puede mejorar el desempeño del sistema de innovación, no genera unos impactos tan marcados en su desempeño.
Por otra parte, se resaltan las características novedosas del modelo: i) ofrece un enfoque market-pull; ii) permite la co-evolución y coespecialización de los agentes; iii) considera un entorno competitivo dinámico que genera múltiples OI en cada período representando diferentes necesidades o atributos; iv) premia a los agentes que suplen las OI, asignando un ciclo de vida a la innovación y una volatilidad a las OI; v) rige la búsqueda de agentes por una lógica de cercanía; vi) se considera a los agentes con una racionalidad limitada; vii) no se limita a los agentes en la búsqueda de colaboradores complementarios distantes; y viii) permite a los agentes tener todo tipo de capacidades, así como su posible acumulación y des-acumulación.

Como trabajo futuro se requiere avanzar en la validación del modelo, tanto desde el punto de vista conceptual, como operacional. De forma similar, solo se analizaron cuatro escenarios que se requerían para dar respuesta al objetivo del trabajo; sin embargo, el modelo posibilita dos tipos de aplicaciones: por un lado, para el desarrollo teórico, en el sentido de Davis, Eisenhardt y Bingham (2007); por otro lado, para la formulación de políticas sobre intermediación y aprendizaje, según se desprenda de la comprensión que genere el modelo sobre el papel de los intermediarios a partir de las simulaciones. Con respecto al modelo, se pueden adicionar nuevas variables y/o relaciones que permitan realizar otros análisis del papel de los intermediarios; estas adiciones pueden estar enfocadas, por ejemplo, a la creación de spin-offs que hereden capacidades que los agentes de origen no están usando, como alternativa al des-aprendizaje; así como a permitir la imitación, donde los agentes puedan competir por medio de esta estrategia por las OI que ya están siendo suplidas, especialmente incorporando el modelo Bass; de forma similar, se puede diferenciar entre enlaces débiles y fuertes que influyan en la topología de red, así como permitir a los agentes priorizar sus vínculos con agentes con quienes ya tuvieron una relación anterior, entre otras posibilidades.

\section{Agradecimientos}

Los autores agradecen a Colciencias, el Departamento Administrativo de Ciencia, Tecnología e Innovación de Colombia, por la beca de estudios doctorales de Walter Lugo Ruiz Castañeda que ha hecho posible el trabajo aquí presentado.

\section{Referencias}

Batterink, M. H., Wubben, E. F., Klerkx, L., \& Omta, S. (2010). Orchestrating innovation networks: The case of innovation brokers in the agri-food sector. Entrepreneurship \& Regional Development , 22 (1), 47-76.

Bertalanffy, L. (1968). General System Theory: Foundations, Development, Applications. New York: George Braziller.

Boon, W. P., Moors, E. H., Nahuis, R., \& Vandeberg, R. L. (2008). User-producer interactions in emerging pharmaceutical and food innovations. International Journal of Innovation Management , 12 (3), 459-487. 
Davis, J., Eisenhardt, K., \& Bingham, C. (2007). Developing Theory through Simulation Methods. Academy of Management Review , 32 (2), 480-499. Dyer, J., \& Singh, H. (1998). The relational view: cooperative strategy and sources of interorganizational competitive advantage. The Academy of Management Review , 23, 660-679.

Edquist, C. (1997). System of Innovation: Technologies, Institutions and Organisations. (C. Edquist, Ed.) London: Pinter/Cassell.

Ernst, D., Mytelka, L., \& Ganiatsos, T. (1998). Technological capabilities in the context of export-led growth. A conceptual framework. En D. Ernst, T. Ganiatsos, \& L. Mytelka (Edits.), Technological Capabilities and Export Success in Asia (págs. 5-45). London and New York: Routledge.

Gilbert, N., Pyka, A., \& Ahrweiler, P. (2001). Innovation Networks - A Simulation Aproach. Journal of Artificial Societies and Social Simulation , 4 (3).

Göktepe, D. (2006). Bridging inventors with industry: a comparative study of technology transfer organizations. theoretical discussion with preliminary research result. Lund, Sweden: Division of Innovation Lund Institute of Technology Sweden.

Guan, J., \& Ma, N. (2003). Innovative capability and export performance of Chinese firms. Technovation, 23, 737-747.

Hobday, M. (1997). Innovation in East Asia. The Challenge to Japan. Cheltenham and Lyme: Edward Elgar.

Holland, J. H. (2004). El Orden Oculto: De cómo la adaptación crea la complejidad. (E. Torres-Alexander, Trad.) México, D.F., México: Fondo de Cultura Económica.

Kim, L. (1997). Imitation to Innovation. The Dynamics of Korea's Technological Learning. Massachusetts: Harvard Business School Press.

Klerkx, L., \& Leeuwis, C. (2008). Matching demand and supply in the agricultural knowledge infrastructure: experiences with innovation intermediaries. Food Policy, 33 (3), 260-276.
Klerkx, L., \& Leeuwis, C. (2009). Establishment and embedding of innovation brokers at different innovation system levels: Insights from the Dutch agricultural sector. Technological Forecasting \& Social Change, 76, 849-860.

Konttinen, J., Nieminen, M., \& Suvinen, N. (2010). How necessary are intermediary organizations in the commercialization of research. European Planning Studies , 18 (9), 1365-1389.

Parjanen, S., Melkas, H., \& Outila, T. (2011). Distances, Knowledge Brokerage and Absorptive Capacity in Enhancing Regional Innovativeness: A Qualitative Case Study of Lahti Region, Finland. European Planning Studies , 19 (6), 921-948.

Rahmandad, H., \& Sterman, J. (2008). Heterogeneity and Network Structure in the Dynamics of Diffusion: Comparing Agent-Based and Differential Equation Models. Management Science , 54 (5), 998-1014.

Resnick, M. (2001). Tortugas, termitas y atascos de tráfico: exploraciones sobre micromundos masivamente paralelos (Vol. 37). (J. A. Álvarez, Trad.) Gedisa.

Rothwell, R. (1994). Towards the Fifth-generation Innovation Process. International Marketing Review, 11 (1), 7-31.

Ruiz, W. L., \& Robledo, J. (2013). Evaluación del Impacto de los Intermediarios en los Sistemas de Innovación: Marco de Análisis. ALTEC XV Congreso Latino-Iberoamericano de Gestión Tecnológica. Porto: ALTEC.

Van Lente, H., Hekkert, M., Smits, R., \& Van Waveren, B. (2003). Roles of systemic intermediaries in transition processes. International Journal of Innovation Management, 7 (3), 1-33.

Wang, C., Lu, I., \& Chen, C. (2009). Evaluating firm technological innovation capability under uncertainty. Technovation, 28, 349-363.

Williamson, O. E. (1985). The Economic Institutions of Capitalism: Firms, Markets, Relational Contrating. New York: The Free Press A Division of Macmillan, Inc. 